\title{
ASO Author Reflections: Adrenocortical Carcinoma-All We Need Are Nodes
}

\author{
J. Tseng, MD, and M. Jain, MD \\ Division of Minimally Invasive and Endocrine Surgery, Department of Surgery, Cedars-Sinai Medical Center, \\ Los Angeles, CA
}

Adrenocortical carcinoma (ACC) is a rare endocrine malignancy for which several key prognostic factors are unknown. $^{1,2}$ It is unclear whether lymphadenectomy is important solely for staging purposes or whether it also improves survival. ${ }^{3}$ Furthermore, a significant unanswered question is whether metastatic lymph node burden is a qualitative or quantitative variable in its effect on prognosis; that is, whether simply the presence of metastatic lymph nodes is prognostic of worse survival, or whether increasing metastatic lymph node burden increasingly affects survival. $^{3}$

Our study utilized the National Cancer Database to evaluate patients with Stage I-III ACC who underwent adrenalectomy. ${ }^{4}$ Of these 1638 patients, only 386 patients $(22.9 \%)$ underwent lymphadenectomy. Patients who underwent lymphadenectomies had significantly improved survival on multivariable Cox regression, suggesting that adrenalectomy with concurrent lymphadenectomy may provide better oncologic control than adrenalectomy alone. We also found that $17.5 \%$ of clinical N0 patients who underwent lymphadenectomy upstaged to pathologic N1 disease. In contrast, only $0.2 \%$ of clinical N0 patients who did not undergo lymphadenectomy upstaged to pathologic N1 disease. Without lymphadenectomy, a significant portion of $\mathrm{pN} 1$ disease would be understaged as $\mathrm{pN} 0$, stressing the importance of performing lymphadenectomies in ACC patients for accurate staging. Finally, we demonstrate that

(C) Society of Surgical Oncology 2021

First Received: 1 November 2021

Accepted: 1 November 2021;

Published Online: 20 November 2021

M. Jain, MD

e-mail: monica.jain@cshs.org overall survival incrementally worsens with increasing quantitative metastatic lymph node burden. This is the first report of quantitative metastatic lymph node burden in ACC as a robust prognostic factor for survival. This could potentially lead to future improvements in the AJCC TNM staging system for ACC, which currently only qualifies lymph node metastasis as a qualitative prognostic factor.

Our data on the importance of assessing lymph node status in ACC underscore the need to create a nationwide research collaborative for ACC. The findings from our retrospective study should be verified with multi-institutional, prospective, randomized clinical trials, which would be difficult to conduct without a national effort due to the rarity of the disease.

DISCLOSURES No authors have any commercial interest in the subject of the study.

\section{REFERENCES}

1. Ayala-Ramirez M, Jasim S, Feng L, Ejaz S, Deniz F, et al. Adrenocortical carcinoma: clinical outcomes and prognosis of 330 patients at a tertiary care center. Eur $J$ Endocrinol. 2013;169:891-9.

2. Kebebew E, Reiff E, Duh QY, Clark OH, McMillan A. Extent of disease at presentation and outcome for adrenocortical carcinoma: have we made progress? World J Surg. 2006;30:872-8.

3. Alanee S, Dynda D, Holland B. Prevalence and prognostic value of lymph node dissection in treating adrenocortical carcinoma: a national experience. Anticancer Res. 2015;35:5575-9.

4. Tseng J, DiPeri T, Chen Y, Shouhed D, Ben-Shlomo A, et al. Adrenocortical carcinoma: the value of lymphadenectomy. Ann Surg Oncol. 2021. https://doi.org/10.1245/s10434-021-11051-5.

Publisher's Note Springer Nature remains neutral with regard to jurisdictional claims in published maps and institutional affiliations. 